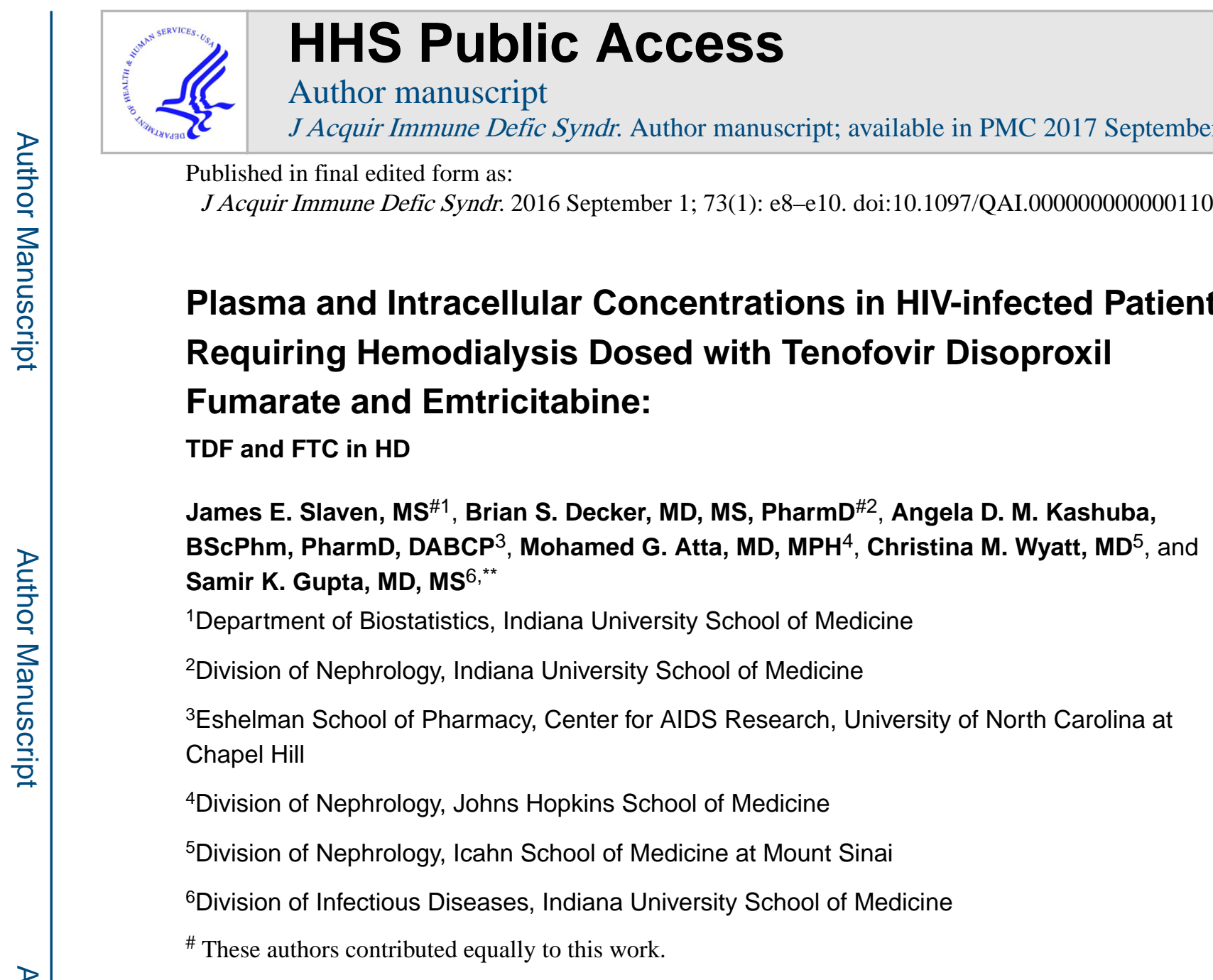

\title{
To the Editors
}

The prevalence of HIV-infected patients requiring chronic hemodialysis (HD) for end stage renal disease (ESRD) continues to increase. ${ }^{1}$ As such, determining the optimal dosing frequency of commonly used antiretrovirals is important to maintain viral suppression while avoiding toxicity. Tenofovir (TFV), administered as TFV disoproxil fumarate (TDF), and emtricitabine (FTC) are renally excreted and require dosing modification for patients with kidney disease. However, the dosing recommendations for these drugs in HD are based on single dose pharmacokinetic studies performed in HIV-uninfected persons with ESRD. ${ }^{2,3}$ Thus, it is important to determine the actual steady-state plasma and intracellular drug concentrations of these drugs for HIV-infected patients on hemodialysis. Understanding the pharmacokinetics of these drugs may also be useful if these agents are to be used by HIVnegative patients on HD as pre-exposure prophylaxis to prevent HIV infection.

As such, we prospectively collected whole blood samples at three clinical sites from $11 \mathrm{HIV}$ infected patients undergoing thrice weekly HD. All participants had been receiving either TDF $300 \mathrm{mg}$ once weekly after HD (n=10) or FTC $200 \mathrm{mg}$ twice weekly after HD $(n=1)$ for at least four weeks to ensure that the measurements reflected steady state. Blood sampling

\footnotetext{
${ }^{* *}$ Corresponding author: Emerson Hall, Suite 421, 545 Barnhill Drive, Indianapolis, IN, 46202, USA; phone 317-274-7926; fax 317-274-1587; sgupta1@iu.edu.

Conflicts of Interest: All other authors, no conflicts.
} 
was performed immediately before HD on the day that the patient was scheduled to take the medication. Plasma TFV and FTC concentrations and peripheral blood mononuclear cell (PBMC) intracellular tenofovir-diphosphate (TFV-DP) and emtricitabine-triphosphate (FTCTP) concentrations were measured using high performance liquid chromatography methods with mass spectrometry detection as previously described. ${ }^{4}$ All participants provided written, informed consent. This study was approved by the respective Institutional Review Boards from the three enrolling sites.

These plasma and intracellular concentrations were then compared to historically reported controls with normal renal function receiving these drugs at steady state (Table 1). ${ }^{5-8}$ One study of TDF given as a single-dose to HIV-uninfected patients on HD was also included for comparison given that this study was used to guide formal dosing recommendations. ${ }^{2} \mathrm{We}$ could not identify any published literature describing the pharmacokinetics of these drugs in HIV-infected patients requiring HD except for a single case report of TDF. ${ }^{9}$ We formally compared our results to these historical controls using geometric mean ratios (GMR), which were then analyzed using two-sided Student's t-tests to determine if the GMR values were significantly different from unity. We also performed non-parametric tests of comparison, which provided similar results (data not shown). All analyses were performed using SAS v9.4 (SAS Institute, Cary, NC) with assumptions being verified at the time of analysis.

The median (range) age and duration of therapy with the medications under study were 56.0 (24.6-65.8) years and 5.0 (3.1-10.5) years, respectively. The median (range) CD4 cell count and HIV-1 RNA level prior to drug initiation were, respectively, $391(153-840) / \mu \mathrm{L}$, and $24,826(2,630-703,800)$ copies/mL. All had HIV-RNA levels $<20$ copies/mL at time of the study visit blood draw. All 11 participants were of self-reported black race, and 9 were men. None had active hepatitis B co-infection, hepatic cirrhosis, or serious illness within 30 days prior to the blood draw.

Due to sample collection and processing issues, plasma concentrations were available from five and PBMC intracellular concentrations from eight of the ten participants receiving TDF. The median (range) TFV plasma concentration was 660.0 (359.0 to 1019.6) ng/mL, with a mean (SD) value of $649.1(266.3) \mathrm{ng} / \mathrm{mL}$. The median (range) TFV-DP intracellular concentration was 317.4 (154.5 to 922.2) fmol/million cells, with a mean (SD) value of 467.5 (333.9) fmol/million cells. The mean TFV plasma and intracellular GMRs were significantly greater than 1 when comparing to the historical groups with normal renal function but less than 1 when comparing to the uninfected historical HD group receiving a single dose of TDF (all $\mathrm{P}<0.05$ ), albeit with substantial overlap in the ranges. Paired plasma and intracellular concentrations were available for three participants; the Spearman correlation between these two measures was $0.50(\mathrm{P}=0.67)$.

The one participant receiving FTC had a plasma concentration of $420 \mathrm{ng} / \mathrm{mL}$, which is appreciably higher than the mean concentration of $57 \mathrm{ng} / \mathrm{mL}$ observed with daily dosing in historical controls with normal renal function. The FTC-TP concentration of $2260 \mathrm{fmol} /$ million cells was similar to the mean concentration of $4000 \mathrm{fmol} / \mathrm{million}$ cells observed in historical controls. 
To our knowledge, this is the first evaluation of tenofovir and emtricitabine steady-state concentrations in HIV-infected patients receiving HD. Even when TDF was given just once per week as recommended for those on HD, we found that TFV plasma and TFV-DP PBMC concentrations were much higher than those previously observed in persons with normal renal function. As higher circulating TFV concentrations may be associated with lower bone mineral density, the plasma TFV concentrations found in our study are potentially concerning. Whether switching TDF to tenofovir alafenamide, which is associated with much lower circulating TFV concentrations and improved bone mineral density, ${ }^{10}$ in patients on HD will result in improved bone mineral density is currently being assessed in an ongoing study (ClinicalTrials.gov NCT02600819). In contrast, the steady-state plasma TFV concentrations in the current study were significantly lower, albeit only modestly in absolute terms, compared to those measured in a single-dose PK study of HIV-uninfected persons receiving HD. This latter finding may not be clinically relevant in terms of virologic control, given the high intracellular TFV-DP concentrations found in our analysis.

Assuming that TFV tissue distribution is similar between HIV-infected and uninfected patients requiring HD, our data suggest that $300 \mathrm{mg}$ of TDF given once weekly may be sufficient to prevent HIV acquisition, at least in uninfected men. In a modeling exercise from the iPrEX data, Anderson et al ${ }^{11}$ found that achieving intracellular TFV-DP concentrations $>16 \mathrm{fmol} /$ million cells was $90 \%$ effective in preventing HIV in men who have sex with men. Because TFV-DP is unstable over time in cryopreserved cells as used in the iPrEx analysis, it is not clear how these data compare to concentrations derived from freshly pelleted and frozen PBMC as done in our study. However, the lowest intracellular level in our TDF group was $154.5 \mathrm{fmol} / \mathrm{million}$ cells, which is appreciably higher than the $16 \mathrm{fmol} / \mathrm{million}$ cell threshold for protection identified in men in iPrEX. It remains to be determined if similar circulating levels are achievable in women requiring HD and would provide equivalent protection against HIV acquisition.

Our steady-state data for the one participant receiving FTC 200mg twice per week suggest that the plasma concentrations of this drug in HD are much higher than those found in patients with normal renal function receiving the same dosage daily. Given that FTC is welltolerated and without known major complications, our finding of a high plasma FTC concentration is likely not of major clinical concern. The intracellular concentration in our participant was similar to the historical control group with normal renal function. Although we were unable to perform statistical comparisons, to our knowledge these are the only available data evaluating steady state FTC levels in patients on HD.

We acknowledge that a full pharmacokinetic analysis delineating the entire concentrationtime curve of these drugs would provide more information regarding drug absorption and elimination. However, this would have entailed expensive and time-consuming procedures over a period of 4 to 7 days. We presumed that the concentrations obtained before dialysis on the day the study drug was administered would allow insight into the likely antiviral effectiveness of the drug over the entire dosing interval. Although the sample size of our study was small, this did not appear to limit the power to find differences in TFV concentrations between our population and historical control groups. Although unintentional, only men were included in these analyses, so we cannot extrapolate these 
findings to women with HIV. Finally, we did not collect detailed information on residual renal function in our participants, and it is possible that plasma concentrations are lower in patients who have recently initiated HD and have greater residual renal function.

In summary, TDF given once weekly as recommended in HIV-infected patients on HD results in steady-state plasma and intracellular PBMC concentrations which are higher than those found in patients with normal renal function who are taking this drug daily. Additional study is required to determine if TDF could potentially be given even less frequently or at lower doses to patients on HD to achieve virologic suppression in those with HIV and to prevent HIV acquisition in those without HIV.

\section{ACKNOWLEDGEMENTS}

We thank the study participants for their generous participation.

Sources of support: This work was supported by an unrestricted research grant from Gilead Sciences, Inc. and the UNC Center for AIDS Research (CFAR P30 AI50410).

Dr. Gupta has received unrestricted grant support from Gilead Sciences, Inc., Janssen Therapeutics, Inc., and Merck \& Co., Inc., consultancy fees from ICON/Oncolys, and travel support to present research findings from Gilead Sciences, Inc. and Bristol-Myers Squibb. The University of North Carolina has received grant support from Gilead to support research conducted by ADMK.

\section{REFERENCES}

1. Schwartz EJ, Szczech LA, Ross MJ, et al. Highly Active Antiretroviral Therapy and the Epidemic of HIV+ End-Stage Renal Disease. J Am Soc Nephrol. 2005; 8:2412-2420. [PubMed: 15987747]

2. Kearney BP, Yale K, Shah J, et al. Pharmacokinetics and dosing recommendations of tenofovir disoproxil fumarate in hepatic or renal impairment. Clin Pharmacokinet. 2006; 11:1115-1124. [PubMed: 17048975]

3. Emtriva Package Insert. 2012.

4. Cottrell ML, Yang KH, Prince HM, et al. A Translational Pharmacology Approach to Predicting HIV Pre-Exposure Prophylaxis Outcomes in Men and Women Using Tenofovir Disoproxil Fumarate+/-Emtricitabine. J Infect Dis. 2016

5. Rousseau FS, Wakeford C, Mommeja-Marin H, et al. Prospective Randomized Trial of Emtricitabine versus Lamivudine Short-Term Monotherapy in Human Immunodeficiency VirusInfected Patients. J Infect Dis. 2003; 11:1652-1658. [PubMed: 14639535]

6. Hawkins T, Veikley W, St Claire RL 3rd, et al. Intracellular pharmacokinetics of tenofovir diphosphate, carbovir triphosphate, and lamivudine triphosphate in patients receiving triplenucleoside regimens. J Acquir Immune Defic Syndr. 2005; 4:406-411. [PubMed: 16010161]

7. Rousseau FS, Kahn JO, Thompson M, et al. Prototype trial design for rapid dose selection of antiretroviral drugs: an example using emtricitabine (Coviracil). J Antimicrob Chemother. 2001; 4:507-513. [PubMed: 11581229]

8. Hoetelmans RM, Marien K, De Pauw M, et al. Pharmacokinetic interaction between TMC114/ ritonavir and tenofovir disoproxil fumarate in healthy volunteers. Br J Clin Pharmacol. 2007; 5:655661. [PubMed: 17610528]

9. Izzedine H, Launay-Vacher V, Jullien V, et al. Pharmacokinetics of tenofovir in haemodialysis. Nephrol Dial Transplant. 2003; 9:1931-1933. [PubMed: 12937248]

10. Sax PE, Wohl D, Yin MT, et al. Tenofovir alafenamide versus tenofovir disoproxil fumarate, coformulated with elvitegravir, cobicistat, and emtricitabine, for initial treatment of HIV-1 infection: two randomised, double-blind, phase 3, non-inferiority trials. Lancet. 2015; 9987:26062615. [PubMed: 25890673] 
11. Anderson PL, Glidden DV, Liu A, et al. Emtricitabine-tenofovir concentrations and pre-exposure prophylaxis efficacy in men who have sex with men. Science translational medicine. 2012; 151:151ra125. 


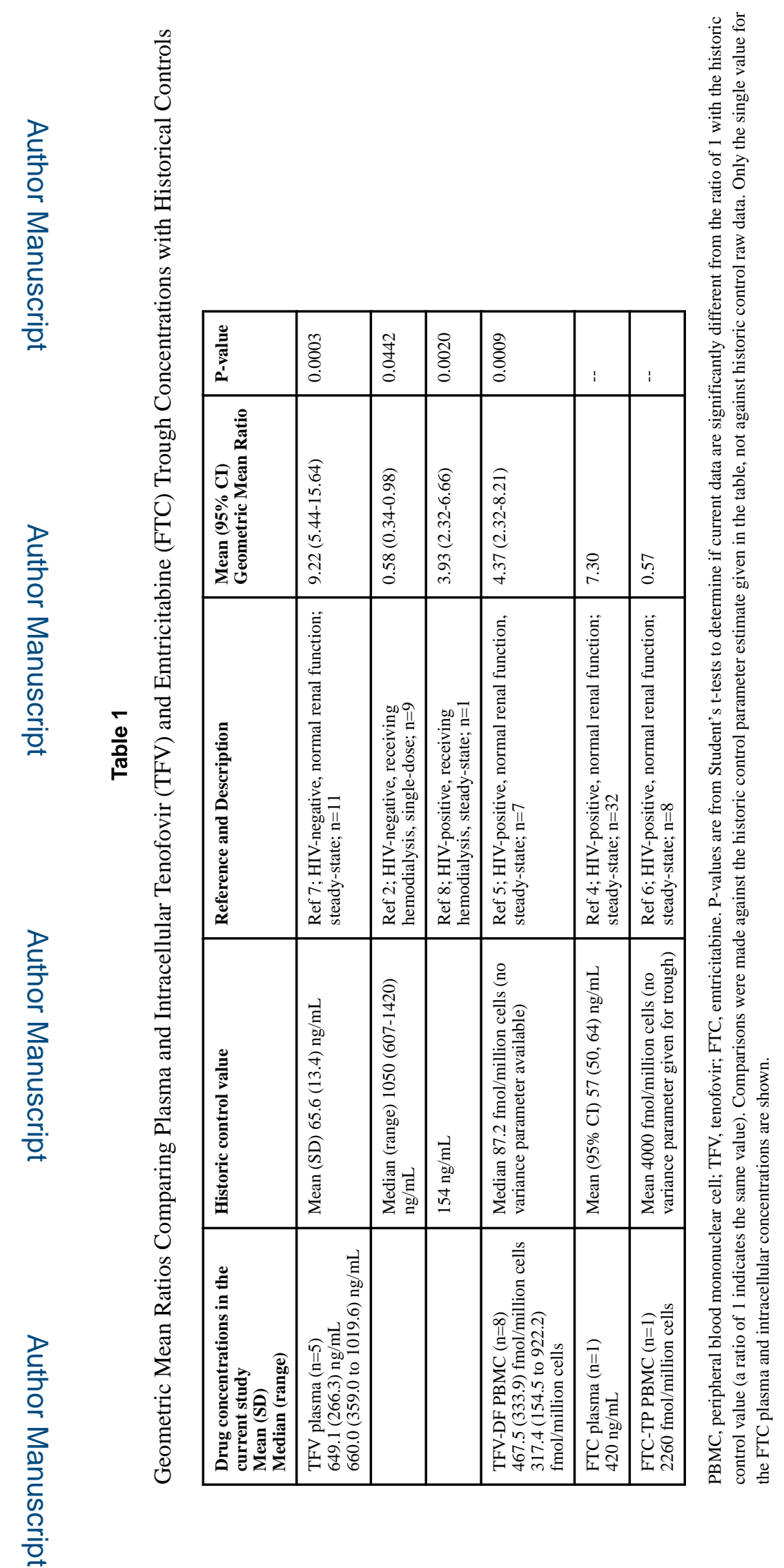

J Acquir Immune Defic Syndr. Author manuscript; available in PMC 2017 September 01. 